\title{
Faint, high proper motion star candidates selected from the SDSS and PS1 catalogs
}

\author{
Noam Segev ${ }^{1}$, Eran O. Ofek ${ }^{\star 1}$ \\ ${ }^{1}$ Benoziyo Center for Astrophysics, Weizmann Institute of Science, 76100 Rehovot, Israel
}

Accepted ? Received ? in original form?

\begin{abstract}
High proper motion stars probe several extreme stellar populations, including nearby objects, Galactic halo stars, and hyper-velocity stars. Extending the search for high proper motion stars, to faint limits can increase their numbers and help to identify interesting targets. We conduct a search for faint $\left(r_{\text {SDSS }}>19.5 \mathrm{mag}\right)$ high proper motion stars $\left(\gtrsim 200 \mathrm{mas} \mathrm{yr}^{-1}\right)$ by comparing the Sloan Digital Sky Survey (SDSS) - Data Release (DR) 10 catalog to the PanSTARRS1-DR1 stacked image catalog. Our main selection criterion is stars that moved $>1$ 1.5 and up to $7^{\prime \prime}$ between the SDSS and PS1 epochs. We identify 2923 high proper motion stars, of which 826 do not have proper motion in the GAIA-DR2 catalog and 565 are not listed in the GAIA-DR2 catalog. Our SDSS-PS1 proper motions are consistent with the GAIA-measured

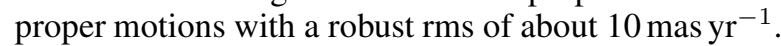

Key words: astrometry - proper motions - stars: binaries: visual - stars: kinematics and dynamics

\section{INTRODUCTION}

High proper motion stars probe several stellar populations. They signal out candidates for nearby objects; probe halo stars and allow us to estimate the halo age using white dwarfs (WD; e.g., Kilic 'et al. 2017); estimate the galactic escape velocity and gravitational potential (e.g., Gnedin et al. 2005; Yu \& Madau 2007, Gnedin et al. 2010; Rossi et al. 2017; Fragione \& Loeb 2017); and discover high-velocity stars, which are presumably formed in three body interactions with the Galactic center black hole (e.g., Brown 2015); as well as identify inter Galactic interlopers (e.g., Erkal et al. 2018; Marchetti, Rossi \& Brown 2018). In addition, the nearest isolated neutron star may hide among faint high proper motion stars (e.g., Ofek 2009). Furthermore, a recent search suggests that some highvelocity stars have unusual spectra (Shen et al. 2018).

The recent GAIA-DR2 release (Gaia Collaboration et al. 2016b; Gaia Collaboration et al. 2018) presents proper motion and parallax fits for about 1.3 billion sources. The GAIA-DR2 catalog still suffers from a high fraction of missing high proper motion stars. This is expected to be improved as more epochs will become available. Tian et al. (2017) presented a proper motion catalog of about $3.5 \times 10^{8}$ stars based on merging the GAIA-DR1 (Gaia Collaboration et al. 2016a), Pan-STARRS1-DR1 (Chambers et al. 2016), SDSS catalog (York et al. 2000), and the 2MASS catalog (Cutri, et al. 2003). Their search is limited to sources that moved up to $1 . .5$ between the SDSS and PS1 epochs (typically $\lesssim 0.2$ mas yr$^{-1}$ ).

\footnotetext{
* e-mail: eran.ofek@weizmann.ac.il
}

Here we present a search for faint $(r$-band mag $>19.5)$ high proper motion objects that moved $>1^{\prime \prime} .5$ and $<7^{\prime \prime}$ between the SDSS and PS1 epochs. Our search is not designed to be complete, and its main purpose is to generate a list of interesting targets for follow-up observations. This source-list may be important for searches of high-velocity stars, cool objects and cool stellar remnants.

In $\$ 2$ we present the search criteria and methodology. In $\$ 3$ we list the candidates and in 4 we discuss the results.

\section{SEARCH FOR PROPER MOTION STARS}

We used the cat s HTM tool (Soumagnac \& Ofek 2018) to query the SDSS-DR10 (Ahn et al. 2014), PS1-DR1 (Chambers et al. 2016; Magnier et al. 2016a; 2016b; 2016c), and GAIA-DR2 (Gaia Collaboration et al. 2016b; Gaia Collaboration et al. 2018) catalogs. We selected SDSS sources that are of type=6 (unresolved); whose angular velocities, as measured in a single SDSS image, are smaller than three times the uncertainty in their velocities (i.e., not an asteroid); and that have $r_{\text {SDSS }}>19.5 \mathrm{mag}$. For each such source, we searched for PS1-DR1 (Chambers et al. 2016; Magnier et al. 2016a; 2016b; 2016c) sources in its neighborhood. We selected SDSS sources that do not have counterparts in the PS1 stackedimage catalog within $1 . .5$. The PS1 stacked-image catalog has the advantage of beeing deeper than single epoch PS1 images. However, due to the several years baseline of the PS1 survey, in many cases proper motion stars are elongated in the stacked image and 
their positional uncertainty is larger than the typical astrometric errors in the catalog.

Next, we searched for PS1 sources around the SDSS source, and selected cases in which there is exactly one PS1 source within $7^{\prime \prime}$ from the SDSS source. We also checked that this PS1 source does not have an SDSS counterpart, within 1.5". Although, this may reject some high proper motion stars (especially at low Galactic latitudes), this step removes a large number of errors and cases of confusion that are hard to verify using only two epochs. Next, we checked that the SDSS $X$-band magnitude is brighter than 21.7 magnitude and that the PS1 $X$-band magnitude is brighter than $22.1 \mathrm{mag}$. Here, $X$-band is either $g$-, $r$-, or $i$-band and we demanded that this criterion is fulfilled for at least one band. We further selected stars for which the best SDSS magnitude error in one of the five SDSS bands is smaller than $0.12 \mathrm{mag}$, and the second best magnitude error is smaller than 0.2 mag. These somewhat arbitrary cuts were very useful in removing large number of problems including spurious sources (e.g., background fluctuations that looks like stars) and image artifacts.

Following this, we selected all SDSS and PS1 sources within $200^{\prime \prime}$ of the candidate position, we matched them by coordinates and calculated the mean offset in right ascension (R.A.) and declination (Dec.) between the two matched lists. If the absolute value of local offset in R.A. was smaller than 0.15 , and the absolute value of local offset in Dec. was smaller than 0.5 , we proceeded; otherwise, we rejected the candidate. This step rejected a large number of false candidates due to bad astrometry.

Next we checked that the $\left|X_{\mathrm{SDSS}}-X_{\mathrm{PS} 1}\right|$ magnitude difference, is smaller than 0.4 mag in at least one of the bands, where $X$ is either $g, r$, or $i$-bands. Finally, we removed sources which have another SDSS source brighter than $r$-band 16 magnitude within $20^{\prime \prime}$, or brighter than $r$-band 13.5 magnitude within $50^{\prime \prime}$. This step rejected large number of fake sources that are found near diffraction spikes and saturated stars.

This selection process yielded 4486 initial candidates for high proper-motion stars. We inspected by eye the SDSS and PS1 images of all the candidates. Sometime, when more than one SDSS image was available, we inspected multiple SDSS images. This inspection revealed many false candidates. The most common problems were due to faint sources that were not identified in one of the images, or sources that are likely due to noise, diffraction spikes and saturated stars, features on top of extended sources, like galaxies and nebulae, that were misidentified as stars, bad astrometry, and transients. All the steps in this search were conducted using tools available as part of the MATLAB Astronomy \& Astrophysics Toolbox 1 (Ofek 2014). After this selection process, we were left with 2923 good candidates for high proper motion stars.

\section{THE HIGH PROPER MOTION CANDIDATES}

Our search yielded 2923 high proper motion candidates, listed in Table 1 We cross-matched these sources with the GAIA-DR2 catalog. Of these 2923, 826 stars do not have proper motion measurements in the GAIA-DR2 catalog, and 565 are not listed in the GAIA-DR2 catalog. 644 stars in this list have SDSS spectra, and their spectral type information is listed in Table 1

Figure 1 presents the candidates' magnitude distribution. To verify our results, we show in Figures 2 and 3 the SDSS-PS1 proper

1 https://webhome.weizmann.ac.il/home/eofek/matlab/

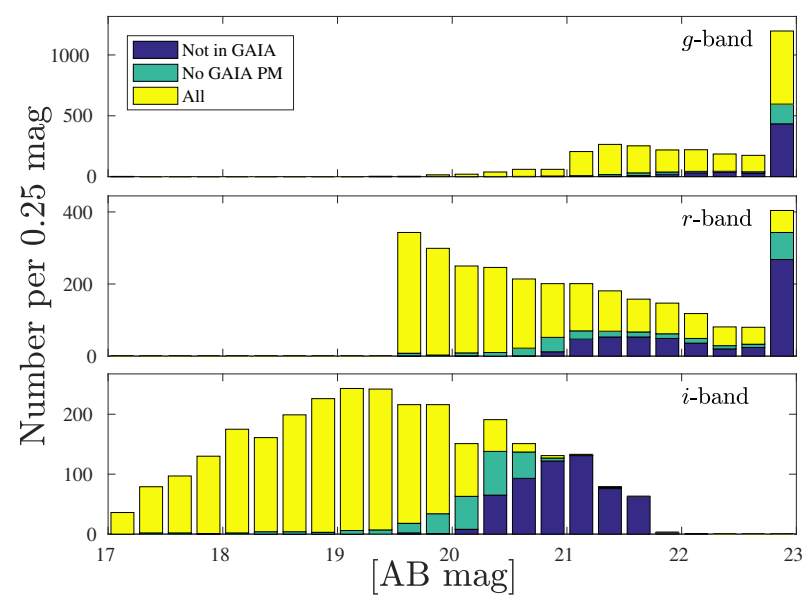

Figure 1. Proper motion star candidates' $g$ (top), $r$ (middle), and $i$ (bottom) SDSS AB-magnitude distribution. The highest-magnitude bin (on the right side) in the $g$-and $r$-bands represents magnitudes fainter than the SDSS detection limit.

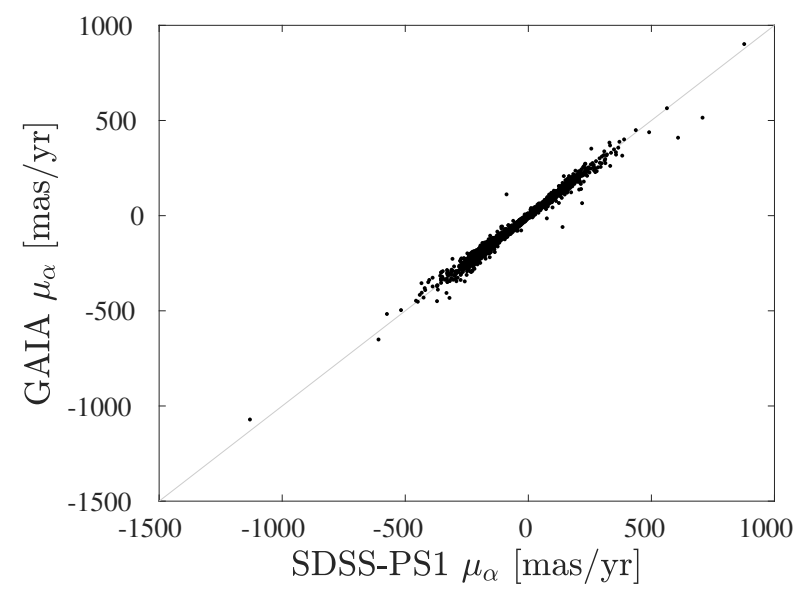

Figure 2. SDSS-PS1 vs. GAIA proper motion in Right Ascension $(\alpha)$ for our high proper motion stars that have proper motion measurements in the GAIA catalog.

motion vs. the GAIA proper motion, in Right Ascension $(\alpha)$ and Declination $(\delta)$, respectively. There is good agreement between the proper motion measurements. Assuming that the GAIA measurement errors are much smaller than our uncertainties (see justification below), these figures also allow us to estimate the uncertainty in our measurements. We find that for the proper motion in rightascension (declination), there is a small bias of about 1 (6) ${\text { mas } \mathrm{yr}^{-1}}^{-1}$ between the SDSS-PS1 and GAIA measurements, and that the scatter is 19 (17) mas $r^{-1}$. This scatter is dominated by a few outliers,

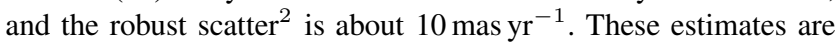
consistent with the expected uncertainty. Specifically, the typical astrometric uncertanty in the PS1 and SDSS images is of the order of 50-100 mas. Adding in quadrature and dividing by the typical time baseline between the two surveys gives expected errors of the

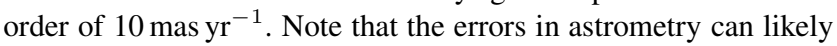
be improved by redoing the astrometry of the PS1 and SDSS images using the GAIA catalog (e.g., Tian et al. 2017).

2 We estimate the robust scatter by calculating the 15.9 to 84.1 percentiles of the distribution divided by two. 


\begin{tabular}{|c|c|c|c|c|c|c|c|c|c|c|c|c|}
\hline \multicolumn{2}{|c|}{ J2000 SDSS } & \multirow[b]{2}{*}{$\begin{array}{c}\mu_{\alpha} \\
\operatorname{mas} \mathrm{yr}^{-1}\end{array}$} & \multirow[b]{2}{*}{$\begin{array}{c}\mu_{\delta} \\
{\text { mas } \mathrm{yr}^{-1}}^{-1}\end{array}$} & \multirow[b]{2}{*}{$\begin{array}{c}\text { Epoch } \\
\text { Julian years }\end{array}$} & \multirow[b]{2}{*}{ Sep. } & \multirow[b]{2}{*}{$\begin{array}{c}\text { Time } \\
\mathrm{yr}\end{array}$} & \multicolumn{6}{|c|}{ SDSS } \\
\hline $\begin{array}{c}\alpha \\
\operatorname{deg}\end{array}$ & $\begin{array}{c}\delta \\
\operatorname{deg}\end{array}$ & & & & & & $\underset{\mathrm{mag}}{g}$ & $\begin{array}{c}\delta g \\
\text { mag }\end{array}$ & $\begin{array}{c}r \\
\text { mag }\end{array}$ & $\begin{array}{c}\delta r \\
\text { mag }\end{array}$ & $\begin{array}{c}i \\
\text { mag }\end{array}$ & $\begin{array}{c}\delta i \\
\text { mag }\end{array}$ \\
\hline 358.1971914 & -20.1561758 & -199.3 & -109.1 & 200 & 1.77 & 7.77 & 24.34 & 0.68 & 22.84 & 0.33 & 19.77 & 0.04 \\
\hline 355.0348332 & -19.6746000 & 144.0 & -119.1 & 2004.951 & 1.82 & 9.71 & 21.46 & 0.06 & 20.13 & 0.03 & 18.32 & 0.01 \\
\hline 344.2284300 & -19.1558072 & 298.0 & -19.4 & 2004.951 & 2.23 & 7.45 & 24.76 & 0.70 & 22.06 & 0.14 & 19.60 & 0.03 \\
\hline 312.6912417 & -15.6465449 & 63.6 & -315.6 & 2004.697 & 2.17 & 6.73 & 22.55 & 0.17 & 20.80 & 0.05 & 18.19 & 0.01 \\
\hline 304.1203511 & -13.1012241 & -4.7 & -292.8 & 2005.441 & 1.77 & 6.03 & 23.68 & 0.57 & 21.88 & 0.17 & 18.61 & 0.02 \\
\hline
\end{tabular}

Table 1. List of candidate faint high proper motion stars. The full table is available electronically. Here we give the first five lines, and first 13 columns. Additional columns available in the electronic version are the PS1 gri magnitudes, a flag indicating if a common proper motion star was detected in the images, number of GAIA matches, GAIA proper motion and parallax, SDSS spectroscopic classification and properties.

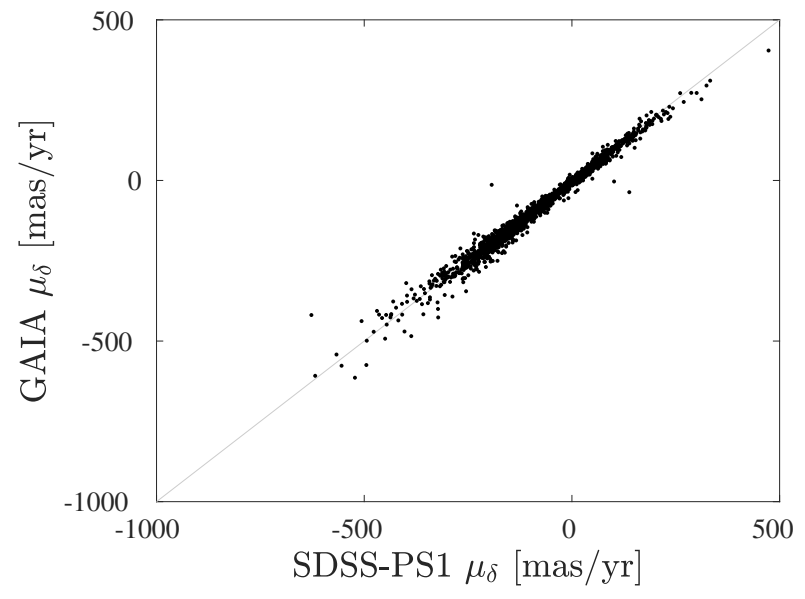

Figure 3. Like Figure2 but for the proper motion in declination $(\delta)$.

\section{DISCUSSION}

We present a non-complete search for faint high proper motion stars from the SDSS and PS1 catalogs. Our search yielded 2923 high proper motion candidates listed in Table 1, of which 826 stars do not have proper motion measurements in the GAIA-DR2 cata$\log$. This is the largest list of high proper motion stars below the USNO-B1.0 (Monet et al. 2003) and GAIA magnitude limits.

Figure 4 shows the SDSS $g-r$ vs. $r-i$ colors of these sources. Red points represent sources with a reduced proper motion of a $H<24$ mag, while black points have $H \geq 24$ mag. Here, the reduced proper motion is defined as

$H=m+5 \log _{10}(\mu)+5=M+5 \log _{10} V-3.379$,

where $M$ is the absolute magnitude, $\mu$ is the total proper motion in arcseconds per year, and $V$ is the transverse velocity in $\mathrm{km} \mathrm{s}^{-1}$. The blue line represents the expected color of a black body source, assuming no reddening. In this plot, hot stars, with an effective temperature above $4000 \mathrm{~K}$, are presumably white dwarfs, while the vertical clump is likely populated by $\mathrm{M}$ dwarfs and brown dwarfs. There are many sources (mainly black points; i.e., $H \geq 24$ ) that are not located on the black-line. These are sources whose magnitude in one of the bands is below the detection limit and, therefore, one of the colors is unreliable.

Figure 5 (6) presents the SDSS $g-r(r-i)$ color of these stars against their reduced proper motion. The right-hand Y-axis shows the transverse velocity assuming an absolute magnitude of 18. Figure 7 (8) shows the mean proper motion in right ascension (declination) as a function of sky location as calculated in 512 hierarchical triangular mesh (Szalay et al. 2007) trixels (triangle side

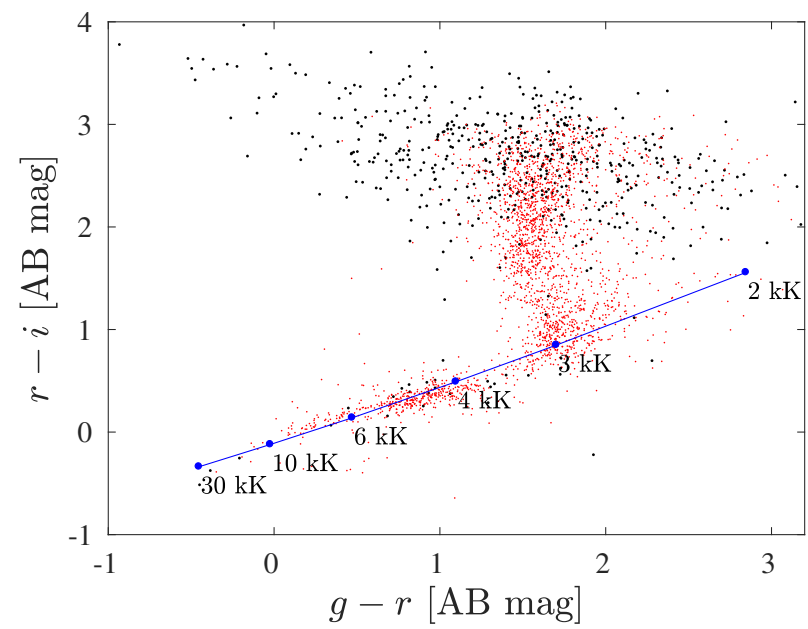

Figure 4. SDSS $g-r$ vs. $r-i$ colors of the high proper motion candidates. Tiny red points represent sources with reduced proper motion of $H<24 \mathrm{mag}$, while black points have $H \geq 24 \mathrm{mag}$. The blue line represent the expected color of a black body source, assuming no reddening. There are many sources (mainly black points) that are not located on the black-line. These are sources whose magnitude in one of the bands is below the detection limit and, therefore, one of the colors is unreliable.

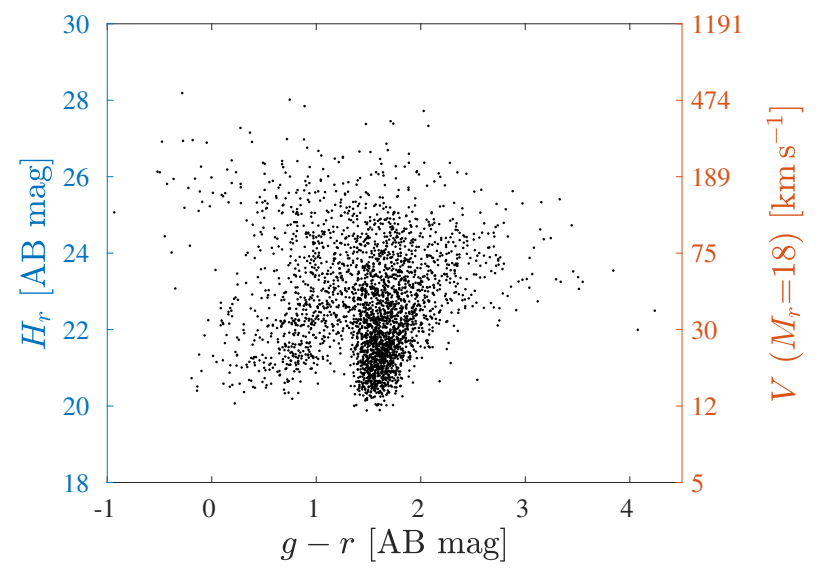

Figure 5. The $g-r$ color vs. reduced proper motion of high proper motion candidates. The right-hand axis shows the transverse velocity assuming absolute $r$-band magnitude of 18 . 

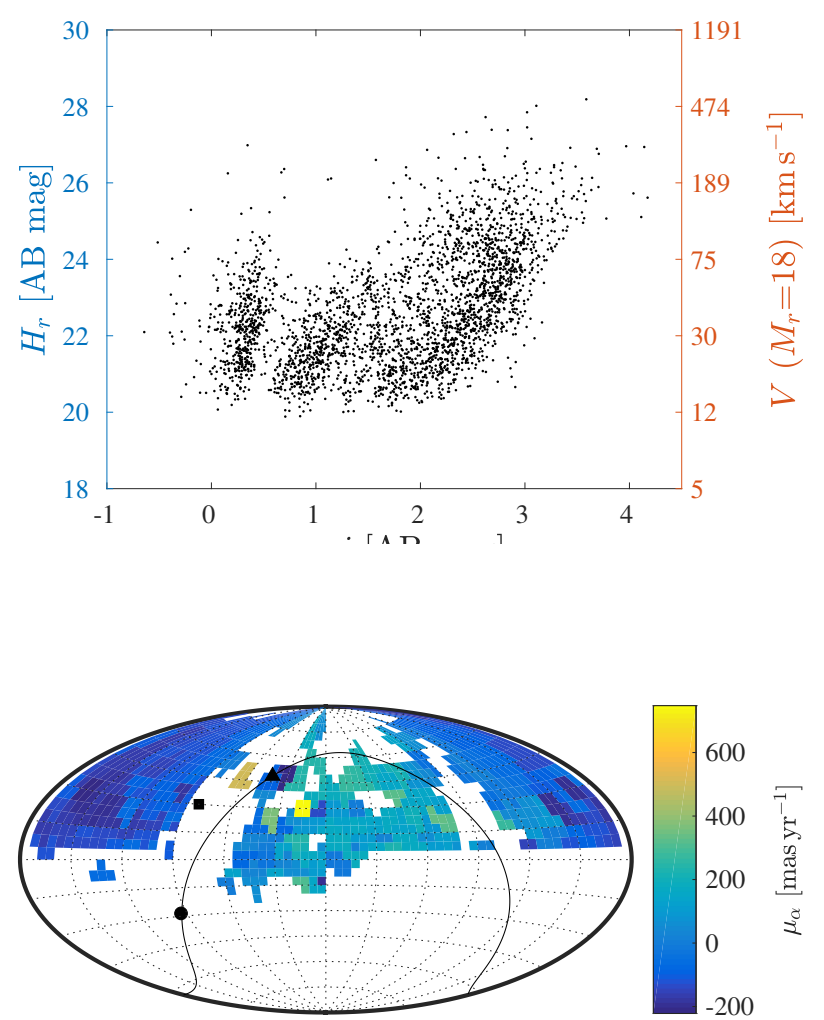

Figure 7. Sky map in Aitoff projection and Equatorial coordinates, of the mean proper motion in Right Ascension in our catalog of 2923 high proper motion stars. The mean proper motion was calculated in 512 hierarchical triangular mesh trixels and then interpolated, using nearest point interpolation, to a regular grid. The solid line represents the Galactic plane, The black circle, triangle, and square, shows the Galactic center, the direction of motion of the solar circle, and the direction of the Solar Apex ( $\alpha=277 \mathrm{deg}$, $\delta=+30 \mathrm{deg}$ ), respectively.

is about $11 \mathrm{deg}$ ). These plots suggest that the proper motion component of faint high proper motion stars are not random and show some structure. Figure 9 presents a quiver map of proper motion, where arrows indicate mean proper motion direction and amplitude, for each trixel in the hierarchical triangular mesh.

An interesting question is what fraction of these new high proper motion stars are high velocity stars, or nearby objects. This question cannot be answered directly. However, we can get an estimated answer by inspecting the $\approx 2000$ stars in our sample for

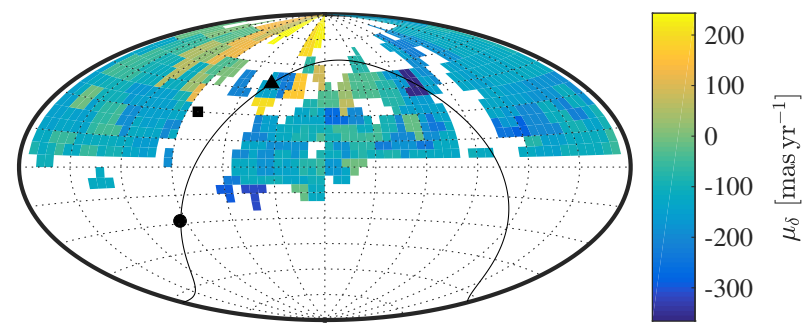

Figure 8. Like Figure 7 but for proper motion in declination.

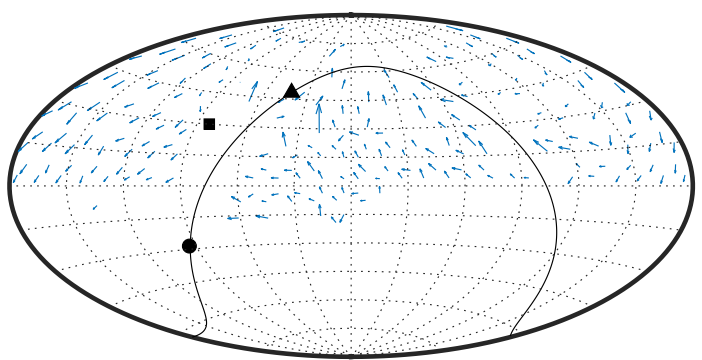

Figure 9. Like Figure 7 but a quiver map of proper motions, where arrows indicate mean proper motion direction and amplitude, for each trixel in the hierarchical triangular mesh.

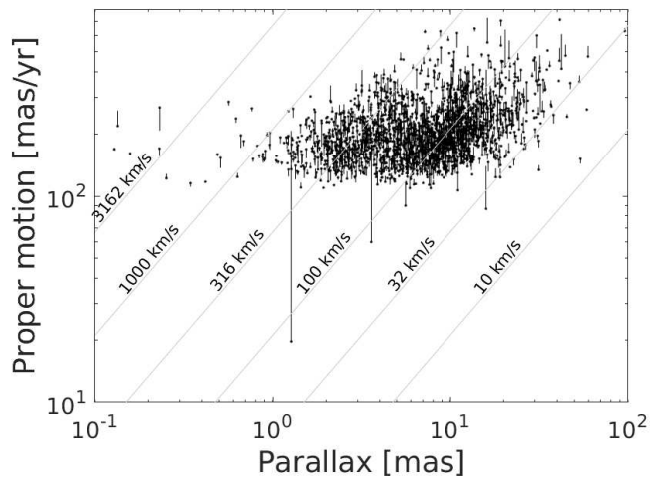

Figure 10. The parallax vs. proper motion of stars in our sample that have GAIA-DR2 proper motions. The difference between a GAIA proper motion measurement and our SDSS/PS1-based measurement is indicated by the vertical lines. The grey curves show lines of equal sky-projected velocities.

and our SDSS/PS1-based measurements are indicated by the vertical lines. The grey curves show lines of equal sky-projected velocities. Presumably most of these faint high proper motion stars are relatively nearby ( $\lesssim 1 \mathrm{kpc}$ ) stars. However, about 30 stars (11 with negative parallaxes and hence are not shown in the plot), have nominal projected velocities consistent with being above $1000 \mathrm{~km} \mathrm{~s}^{-1}$. However, the median error in GAIA parallax is about 0.5 mas, and we cannot rule out that most of the these high velocities are due to errors. Therefore, we estimate that up to about $2 \%$ of the stars in our sample may have projected velocities above about $1000 \mathrm{~km} \mathrm{~s}^{-1}$.

To summarize, our new list of faint proper motion stars contains candidates for hyper velocity stars, halo stars, cool stars, and nearby objects. This list provide an order of magnitude increase in the number of known high proper motion stars fainter than $r$-band magnitude $\approx 20.5$.

\section{ACKNOWLEDGMENTS}

E.O.O. is grateful for the support by grants from the Israel Science Foundation, Minerva, Israeli Ministry of Technology and Science, the US-Israel Binational Science Foundation, Weizmann-UK, and the I-CORE Program of the Planning and Budgeting Committee and the Israel Science Foundation. 


\section{REFERENCES}

Ahn, C. P., Alexandroff, R., Allende Prieto, C., et al. 2014, ApJS, 211, 17

Brown, W. R. 2015, ARA\&A, 53, 15

Chambers, K. C., Magnier, E. A., Metcalfe, N., et al. 2016, arXiv: 1612.05560

Cutri, R. M., Skrutskie, M. F., van Dyk, S., et al. 2003, VizieR Online Data Catalog, II/246.

Erkal, D., Boubert, D., Gualandris, A., Evans, N. W., \& Antonini, F. 2018, arXiv:1804.10197

Fragione, G., \& Loeb, A. 2017, New Astronomy, 55, 32

Gaia Collaboration, Brown, A. G. A., Vallenari, A., et al. 2016a, A\&A, 595, A2

Gaia Collaboration, Prusti, T., de Bruijne, J. H. J., et al. 2016b, A\&A, 595, A1

Gaia Collaboration, Brown, A. G. A., Vallenari, A., et al. 2018, arXiv:1804.09365

Gnedin, O. Y., Gould, A., Miralda-Escudé, J., et al. 2005, ApJ, 634, 344

Gnedin, O. Y., Brown, W. R., Geller, M. J., et al. 2010, ApJ, 720, L108

Kilic, M., Munn, J. A., Harris, H. C., et al. 2017, ApJ, 837, 162

Magnier, E. A., Sweeney, W. E., Chambers, K. C., et al. 2016a, arXiv:1612.05244

Magnier, E. A., Chambers, K. C., Flewelling, H. A., et al. 2016b, arXiv: 1612.05240

Magnier, E. A., Schlafly, E. F., Finkbeiner, D. P., et al. 2016c, arXiv:1612.05242

Marchetti, T., Rossi, E. M., \& Brown, A. G. A. 2018, MNRAS, 2466

Monet, D. G., Levine, S. E., Canzian, B., et al. 2003, AJ, 125, 984

Ofek, E. O. 2009, PASP, 121, 814

Ofek, E. O. 2014, Astrophysics Source Code Library, ascl:1407.005

Rossi, E. M., Marchetti, T., Cacciato, M., et al. 2017, MNRAS, 467, 1844

Shen, K. J., Boubert, D., Gänsicke, B. T., et al. 2018, arXiv:1804.11163

Soumagnac, M. T., \& Ofek, E. O. 2018, arXiv:1805.02666

Szalay, A. S., Gray, J., Fekete, G., et al. 2007, arXiv:cs/0701164

Tian, H.-J., Gupta, P., Sesar, B., et al. 2017, ApJS, 232, 4

York, D. G., Adelman, J., Anderson, J. E., Jr., et al. 2000, AJ, 120, 1579

Yu, Q., \& Madau, P. 2007, MNRAS, 379, 1293 